\title{
COVID-19-associated immune thrombocytopenia
}

\author{
Mara-Andrada Plesu', Gabriel Cristian Bejan², Ioana Veronica Grajdeanu², \\ Anca Angela Simionescu ${ }^{3,4}$, Ana Maria Alexandra Stanescu² \\ ${ }^{1}$ Private Medical Practice Dr. Ioana Veronica Grajdeanu, Bucharest, Romania \\ ${ }^{2}$ Department of Family Medicine, \\ "Carol Davila" University of Medicine and Pharmacy, Bucharest, Romania \\ ${ }^{3}$ Department of Obstetrics and Gynecology, \\ "Carol Davila" University of Medicine and Pharmacy, Bucharest, Romania \\ ${ }^{4}$ Filantropia Clinical Hospital, Bucharest, Romania
}

\begin{abstract}
The coronavirus disease 2019 (COVID-19) is a contagious respiratory tract infection caused by the betacoronavirus SARS-CoV-2. The World Health Organization declared the COVID-19 outbreak a pandemic on March 11, 2020. Since the COVID-19 pandemic started, more than 166 million patients have been tested positive worldwide with more than 3.4 million related death recorded. COVID-19 has a wide range of signs and symptoms. Hematological changes such as lymphopenia, thrombocytopenia, and coagulation disturbances are not unusual in patients with COVID-19. However, the mechanisms causing these changes are partially comprehended. Immune thrombocytopenia was identified to be among the hematologic autoimmune diseases seen in patients infected with SARS-CoV-2. This review summarizes the evidence on COVID-19-associated immune thrombocytopenia and the underlying mechanisms involved in its development.

Keywords: thrombocytopenia, immune thrombocytopenic purpura, COVID-19,
\end{abstract} SARS-CoV-2, coronavirus

\section{INTRODUCTION}

The coronavirus disease 2019 (COVID-19) is a contagious respiratory tract infection caused by the beta-coronavirus SARS-CoV-2. It was first identified in Wuhan, the largest metropolitan area in China's Hubei Province, in late 2019, when a series of pneumonia cases of unknown cause emerged (1). The World Health Organization declared the COVID-19 outbreak a pandemic on March 11, 2020 (2). Since the COVID-19 pandemic started, more than 166 million patients have been tested positive worldwide with more than 3.4 million related death recorded (3).

SARS-CoV-2 infection tends to affect people of all ages, but the clinical manifestations vary depending on their age. Many infections, primarily in children and young adults, are asymptomatic or mild, but severe illness, respiratory failure, and death are more frequent in the elderly and/or people with comorbidities (4).

COVID-19 has a wide range of signs and symptoms. The most common symptoms at onset of illness are fever, fatigue and cough, but also myalgia, anorexia and in severe cases dyspnea. Headache, dizziness, sore throat, and chest pain are less common symptoms, as are gastrointestinal symptoms such as abdominal pain, diarrhea, nausea, and vomiting (4-6). Also, a significant number of patients complained of olfactory and taste disturbances. Anosmia and ageusia are nonspecific symptoms that may be the first or only sign of the condition $(7,8)$.

Most patients reported symptoms of disease, after an incubation period of 1-14 days (typically about 5 days); dyspnea and pneumonia occurred within a median of 8 days from disease onset (8). 
Hematological changes such as lymphopenia, thrombocytopenia, and coagulation disturbances are not unusual in patients with COVID-19. However, the mechanisms causing these changes are partially comprehended (9).

Changes in immune system functions have recently been attributed to the SARS-CoV-2 infection. These changes could range from an abnormal immune response and excessive cytokine production to immune system hyperactivation and a significant increase in immune-inflammatory variables. Autoimmunity and cytokine storm may result from these excessive immune responses $(10,11)$.

Furthermore, studies have identified many cases of COVID-19 patients who developed autoimmune events such as antiphospholipid syndrome, autoimmune cytopenia, Guillain-Barré syndrome and Kawasaki disease. These results suggest that SARS-CoV-2 infection is linked to the development of autoimmune diseases $(11,12)$.

Several hematologic autoimmune conditions have also been identified and shown to complicate COVID-19 management. Immune thrombocytopenic purpura (ITP), autoimmune hemolytic anemia (AIHA), thrombotic thrombocytopenic purpura (TTP), antiphospholipid syndrome (APLS), autoimmune neutropenia, and Evans syndrome were identified to be among the hematologic autoimmune diseases seen in patients infected with SARS-CoV-2 $(11,13)$.

This review summarizes the evidence on COVID19-associated immune thrombocytopenia and the underlying mechanisms involved in its development, based on the PubMed database.

\section{MECHANISMS OF THROMBOCYTOPENIA IN COVID-19}

The novel coronavirus disease has been linked to thrombocytopenia, which may have various mechanisms of occurrence and it may appear at any time during the disease course (14).

Coronaviruses can infect bone marrow cells, triggering abnormal hematopoiesis. Using various receptors, SARS-CoV-2 inhibits hematopoiesis in the bone marrow, resulting in reduced primary platelet formation and thrombocytopenia. Hemophagocytic lymphohistiocytosis is one of the secondary pathways that lead to the destruction of bone marrow progenitor cells. This is caused by excessive proliferation and activation of the mononuclear macrophage system, which results in a cytokine storm $(14,15)$.
SARS-CoV-2 also induces thrombocytopenia by increasing levels of autoantibodies and immune complexes, which causes the immune system to specifically destroy thrombocytes. Platelets with specific antigens can be coated by anti-platelet antibodies and immune complexes, resulting in immune-mediated destruction in the peripheral blood (ie. immune thrombocytopenia - ITP) (14).

Lung injury occurs as a consequence of viral infection and inflammation. Damaged lung tissues and pulmonary endothelial cells can stimulate platelets in the lungs, inducing aggregation and the formation of microthrombi, which enhances platelet consumption $(14,16)$.

\section{COVID-19-ASSOCIATED IMMUNE THROMBOCYTOPENIA}

Immune thrombocytopenic purpura (ITP) is an uncommon autoimmune condition marked by platelet counts below 100,000 platelets per microliter and increased bleeding risk (17). Patients who exhibit thrombocytopenia without a known underlying cause are diagnosed with primary ITP. ITP caused by other disorders (autoimmune diseases, lymphoproliferative disorders), infectious agents or medications is known as secondary ITP (18). Environmental and genetic predisposition have been identified as risk factors for ITP. Among the environmental factors involved in the occurrence of autoimmune thrombocytopenia, several infectious agents are associated with the appearance of this condition, especially in children (eg Helicobacter pylori, hepatitis C virus, human immunodeficiency virus, cytomegalovirus, Epstein Barr virus, and other viruses) (19).

Molecular mimicry is one of the proposed mechanisms by which infections contribute to autoimmunity. To evade the immune response, viral proteins mimic platelet receptors. Cross reactivity against platelet receptors can arise as a result of an immune reaction to these viral proteins, resulting in autoantibodies specific for both the viral protein and platelet receptors $(19,20)$.

Thrombocytopenia is among the most challenging disease entities for physicians to deal with on a daily basis. Mild thrombocytopenia has been reported in approximately $5-10 \%$ of patients with symptomatic SARS-CoV-2 infection (21). Other studies showed that nearly $36 \%$ of COVID-19 patients were found to have moderate thrombocytopenia (22). Deep throm- 
bocytopenia, on the other hand, is uncommon and is linked to disease severity (23). Huang et al. noted that COVID-19 non-survivors (20\%) were more thrombocytopenic than COVID-19 survivors (1\%) (24), while Tang et al. showed that thrombocytopenia is a negative prognostic factor during SARS-CoV-2 infection (25).

Several case reports of isolated thrombocytopenia linked to COVID-19 have recently been described (Table 1). Zulfiqar et al. published the first single case report indicating that COVID-19 infection could be linked to immune thrombocytopenia. He described a case of "de novo" ITP in a 65-year-old woman with COVID-19 who underwent a multi-drug treatment, including intravenous immunoglobulin (IVIG), prednisolone, and eltrombopag, to recover completely (26).

Similar to this case (Patient \#1), additional eleven patients (Patients \#2, \#3, \#4, \#5, \#6, \#10, \#11, \#13, $\# 14, \# 15$, \#16) had previous fever or respiratory symptoms that started 4 days to 4 weeks before admission (26-36). Only two patients (Patients \#7, \#8) were diagnosed with ITP and had no COVID-19 signs or symptoms. In these patients, Ahmed et al. pointed to thrombocytopenia as an early manifestation of the disease, without any other COVID-19 specific symptoms. Both patients presented with haemorrhagic manifestations and severe thrombocytopenia, which responded immediately to intravenous immunoglobulin with a persistent response over time (16).

The preponderance of the cases described ( $\mathrm{Pa}-$ tients \#2, \#3, \#4, \#7, \#8, \#9, \#12, \#13, \#16) had thrombocytopenia with evident signs of bleeding upon admission $(16,27,28,32-33,36)$. In some other eight cases, thrombocytopenia was identified significantly later (Patients \#1, \#5, \#6, \#9, \#10, \#11, \#14 and \#15) $(16,26,28,29,30,31,34,35)$. The majority of critically ill patients develop thrombocytopenia later in the course of the disease, and was associated with a longer hospital stay and a higher mortality (Patients \#5, \#6, \#9, \#11 and \#14) $(16,28,29,31,34)$. In a retrospective analysis, Chen et al. reported that COVID19-associated delayed-phase thrombocytopenia developed in 11.8 percent of enrolling patients and that it is more likely to develop in elderly patients or patients with a low lymphocyte count on admission (37).

The reported nadir platelet counts among the patients diagnosed with COVID-19-associated immune thrombocytopenia were extremely variable, ranging from 23000 cells/L (Patient \#11) to 0 cells/l (Patient \#7) $(29,31)$.

Most patients with bleeding symptoms reported manifestations such as epistaxis, purpuric and petechial rash, ecchymoses, or mucosal bleeding, according to our findings. Only 3 patients out of 16 suffered from intracerebral bleeding (28), intraventricular haemorrhage (31) and subarachnoid haemorrhage (26), one out of which died.

Diagnosing COVID-19-associated immune thrombocytopenia might be challenging because of various other possible causes, such as coagulation activation by COVID-19 infection leading to disseminated intravascular coagulation and subsequent thrombocytopenia. Also, medications used for treating COVID-19, including heparin, azithromycin, and hydroxychloroquine, may trigger thrombocytopenia (38). In the case report published by Zulfiqar et al., the patient had used amoxicillin-clavulanic acid and low-molecular-weight-heparin before the detection of thrombocytopenia, which might also be contributing to the decreasing of the platelet count (26).

The diagnosis of ITP is based on the patient's past medical history, systemic physical examination, and an extensive hematological workup that includes a complete blood count and a peripheral blood smear. To rule out viral-mediated thrombocytopenia, patients should be evaluated for HCV and HIV. In situations of isolated thrombocytopenia, bone marrow analysis is not indicated unless there is a suspicion for aplastic anemia, leukemia, or myelodysplastic syndrome (39).

ITP therapy seeks to minimize severe bleeding by ensuring a balanced platelet count. Treatment for COVID-19-related ITP might be complicated. Intravenous immunoglobulin, glucocorticoids, and thrombopoietin receptor agonists are all commonly used and effective treatments. IVIG is usually designated for individuals with ITP who require a quick rise in platelet levels. IVIG has the liability of not being curative and of being poorly tolerated (40). Because IVIG suppresses macrophage phagocytic functions, it may be effective in treating COVID-19 infection at an incipient phase $(19,41)$.

Concerning the management of the documented cases, medications used were: steroids (prednisone, dexamethasone, and methylprednisone), intravenous immunoglobulin (IVIG), thrombopoietin receptor agonists (TPO-RA e.g. Romiplostim and Eltrombopag), 


\begin{tabular}{|c|c|c|c|c|c|c|c|}
\hline 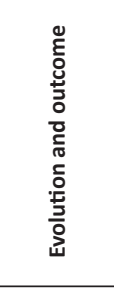 & 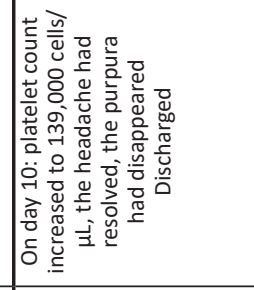 & 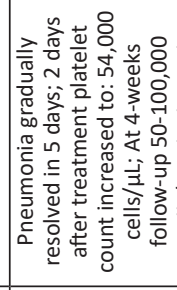 & 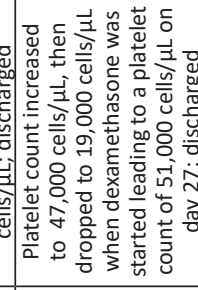 & 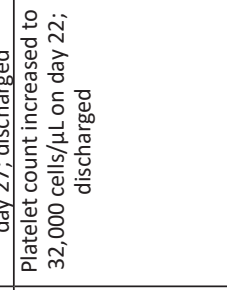 & 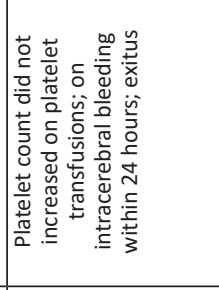 & 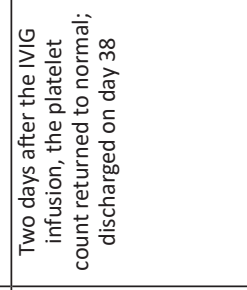 & 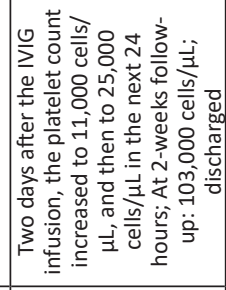 \\
\hline 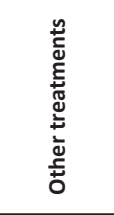 & 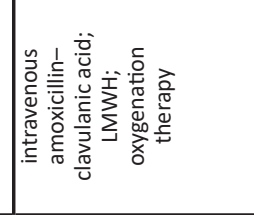 & 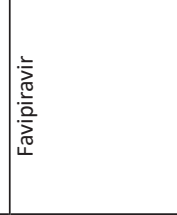 & $\frac{\mathscr{c}}{z}$ & $\frac{\kappa}{2}$ & 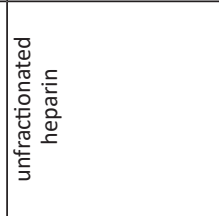 & 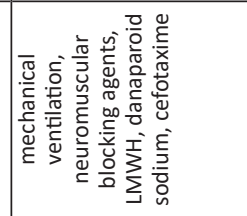 & 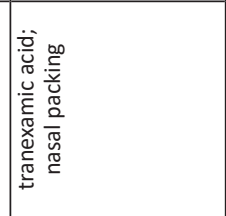 \\
\hline 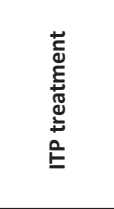 & 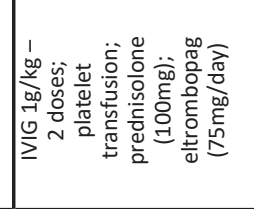 & 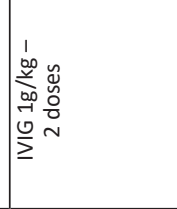 & 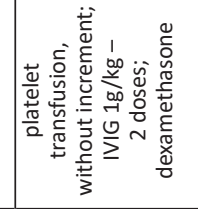 & 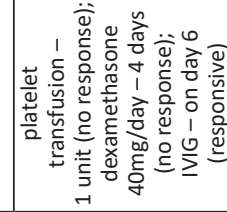 & $\frac{\sqrt{5}}{20}$ & 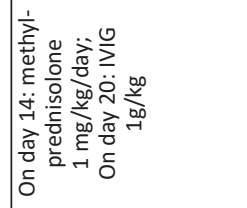 & 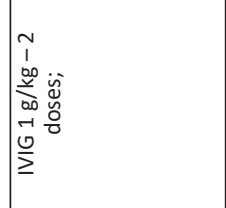 \\
\hline 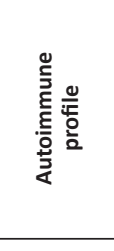 & 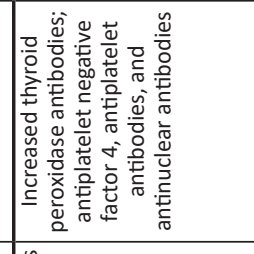 & 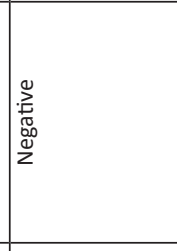 & 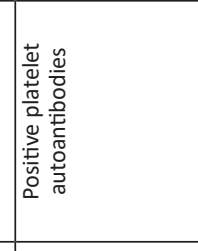 & 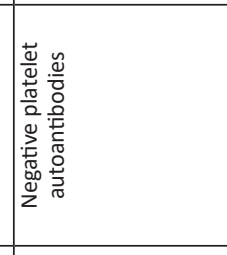 & $\frac{\alpha}{z}$ & 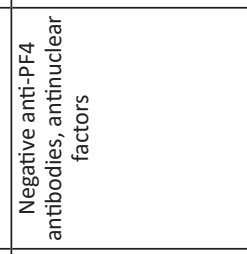 & 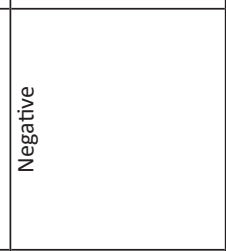 \\
\hline 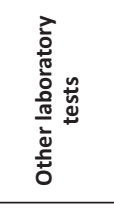 & 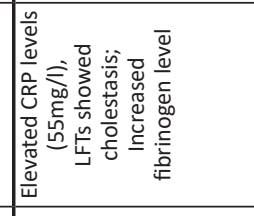 & 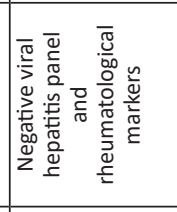 & $\frac{\mathscr{c}}{z}$ & $\frac{\mathscr{c}}{z}$ & $\frac{\kappa}{2}$ & 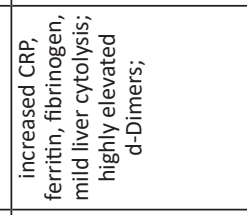 & 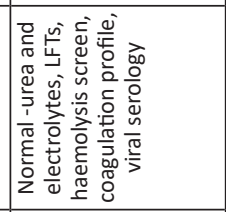 \\
\hline 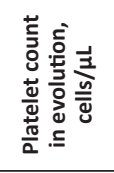 & 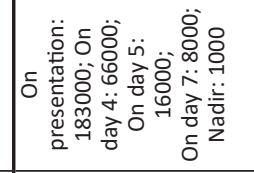 & 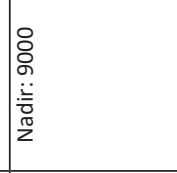 & 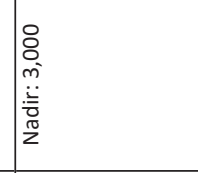 & 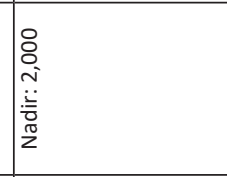 & 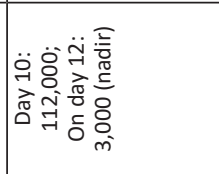 & 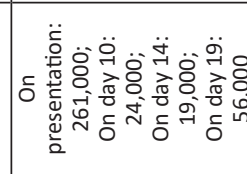 & 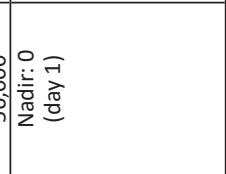 \\
\hline 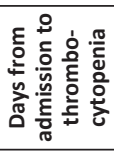 & . & 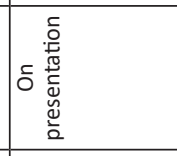 & 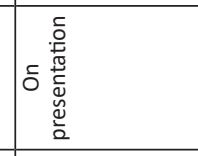 & 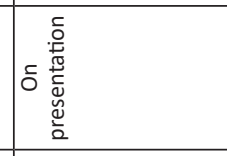 & $\sigma$ & $\infty$ & 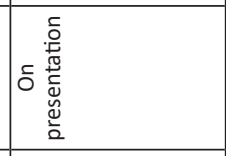 \\
\hline 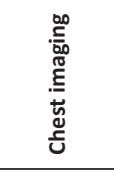 & 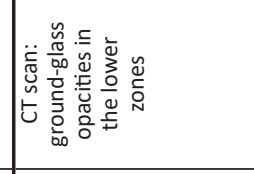 & 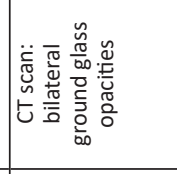 & $\frac{\mathscr{c}}{z}$ & $\frac{\alpha}{z}$ & 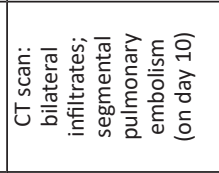 & 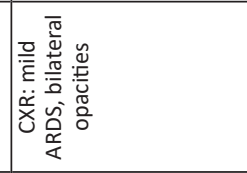 & \begin{tabular}{|c|}
$\overline{\widetilde{\pi}}$ \\
$\underline{\tilde{c}}$ \\
$\check{\partial}$ \\
$\ddot{\tilde{x}}$ \\
$\bar{x}$
\end{tabular} \\
\hline 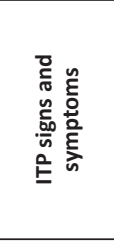 & 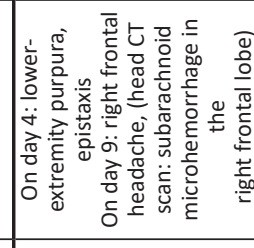 & 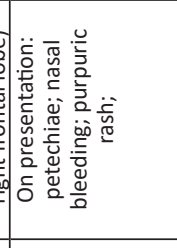 & 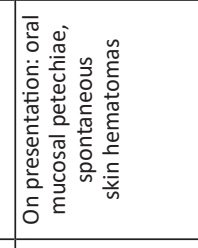 & 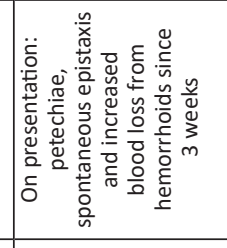 & 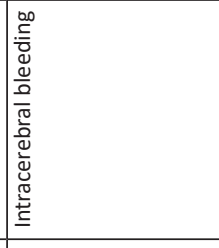 & 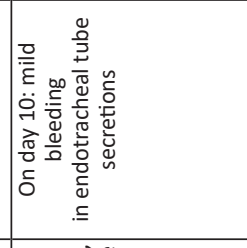 & 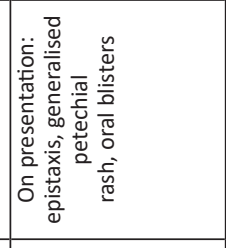 \\
\hline 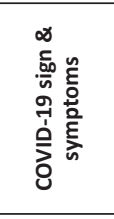 & 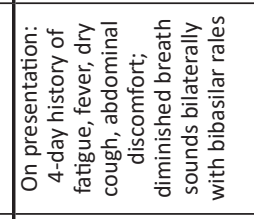 & 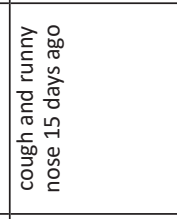 & 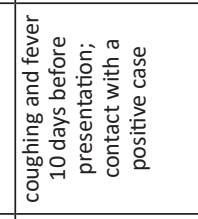 & 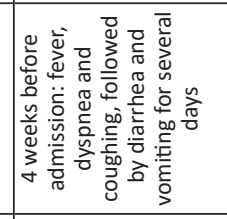 & 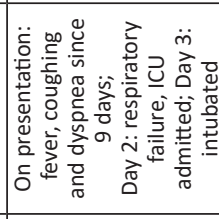 & 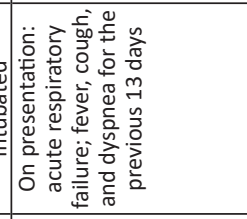 & 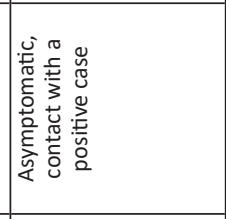 \\
\hline 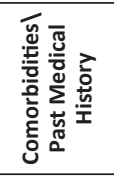 & 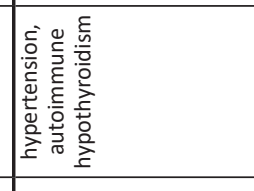 & 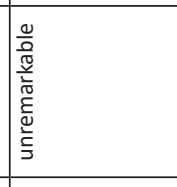 & 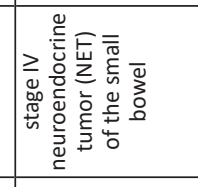 & 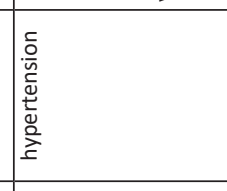 & 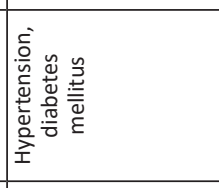 & 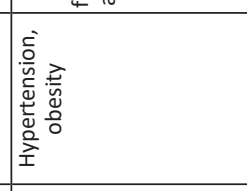 & 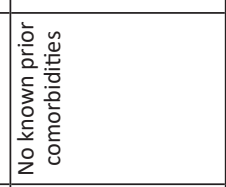 \\
\hline 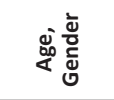 & 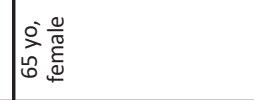 & 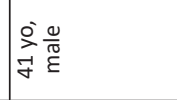 & 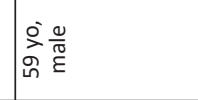 & 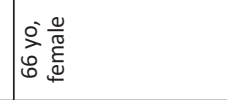 & 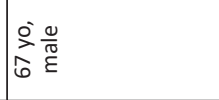 & 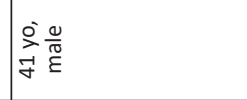 & 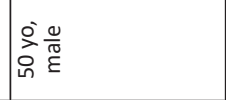 \\
\hline 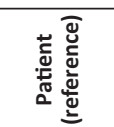 & 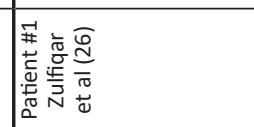 & 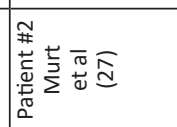 & 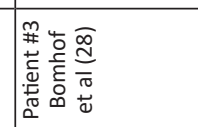 & 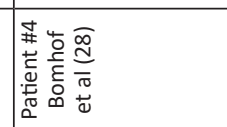 & 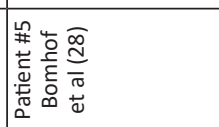 & 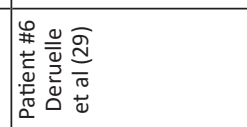 & 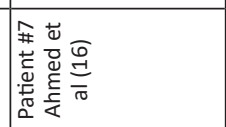 \\
\hline
\end{tabular}




\begin{tabular}{|c|c|c|c|c|c|c|}
\hline 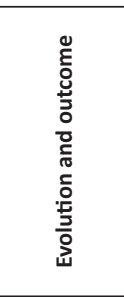 & 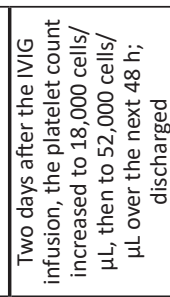 & 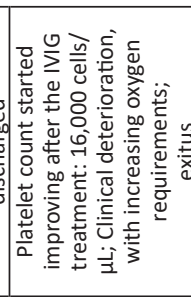 & 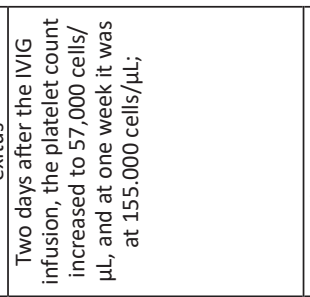 & 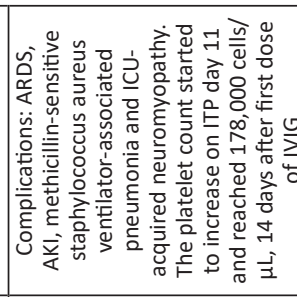 & 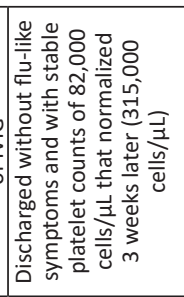 & 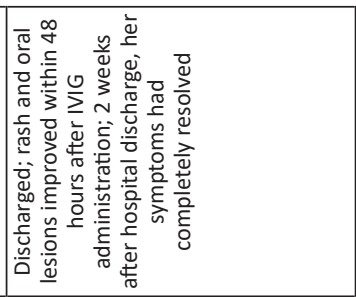 \\
\hline 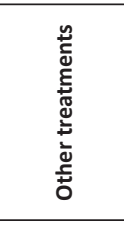 & $\frac{x}{z}$ & 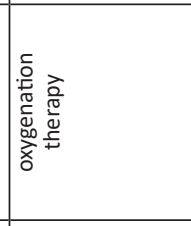 & 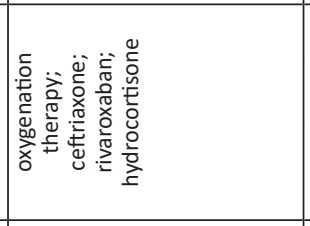 & 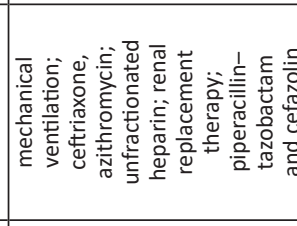 & 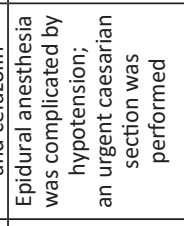 & 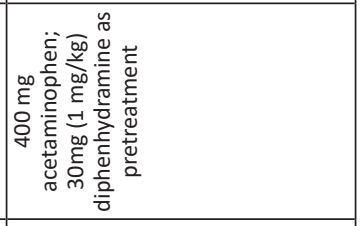 \\
\hline 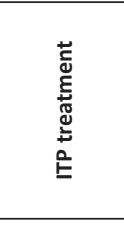 & 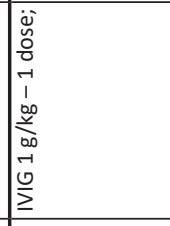 & 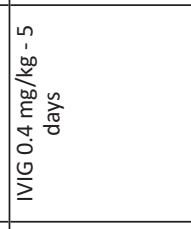 & 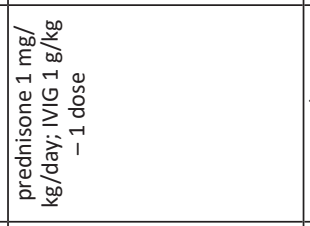 & 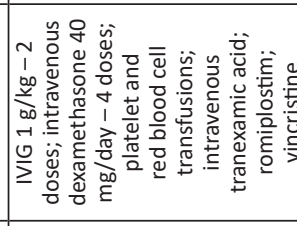 & 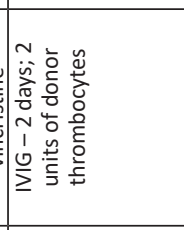 & 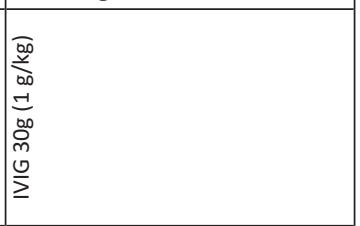 \\
\hline 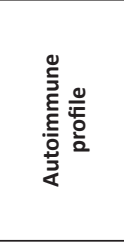 & 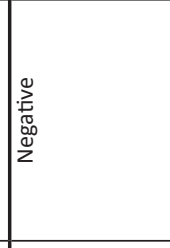 & $\frac{\kappa}{z}$ & 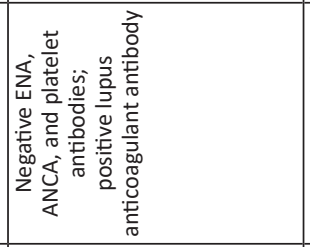 & 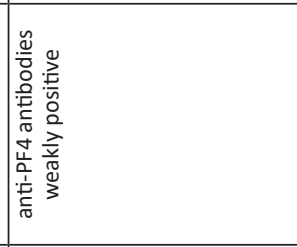 & 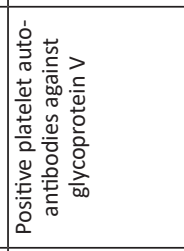 & 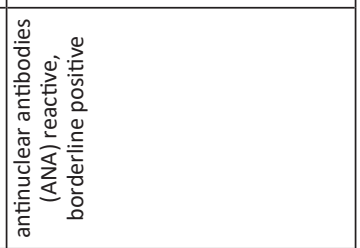 \\
\hline 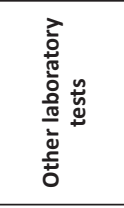 & 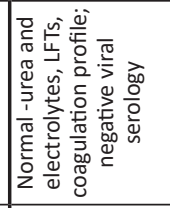 & 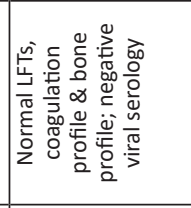 & 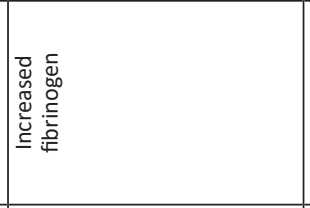 & 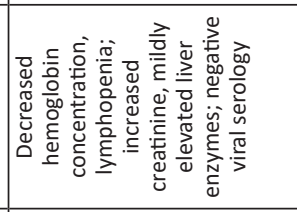 & $\frac{\infty}{2}$ & 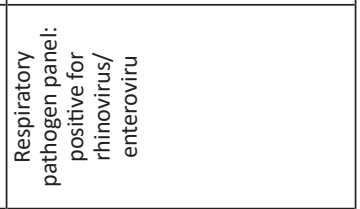 \\
\hline 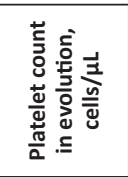 & 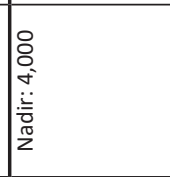 & 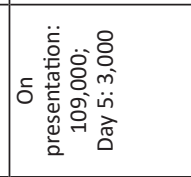 & 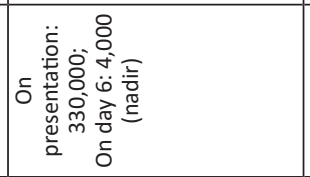 & 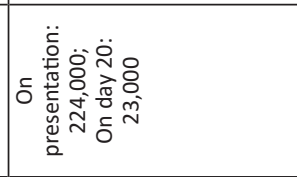 & 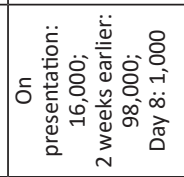 & 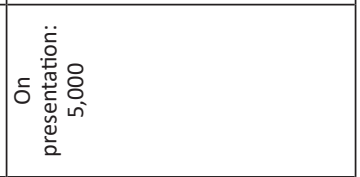 \\
\hline 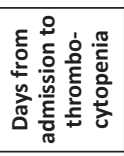 & 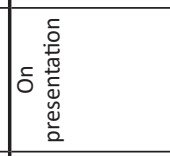 & in & 0 & i & 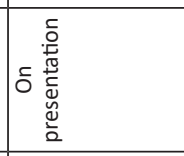 & 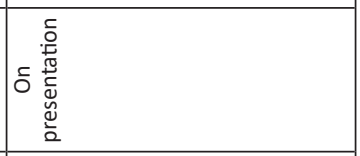 \\
\hline 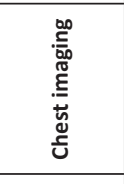 & 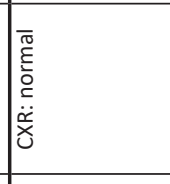 & 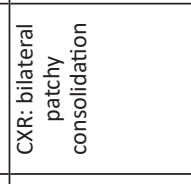 & 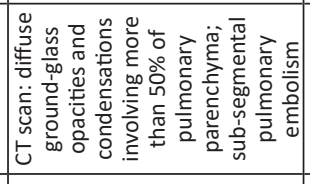 & $\frac{x}{2}$ & 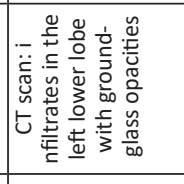 & $\frac{\kappa}{z}$ \\
\hline 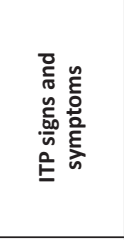 & 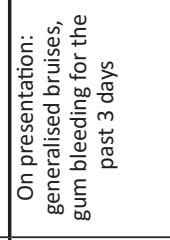 & $\frac{\kappa}{z}$ & 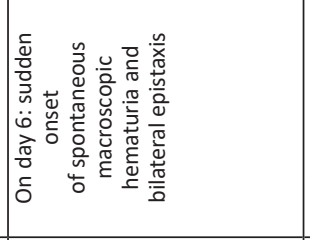 & 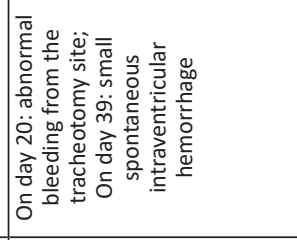 & $\frac{\infty}{z}$ & 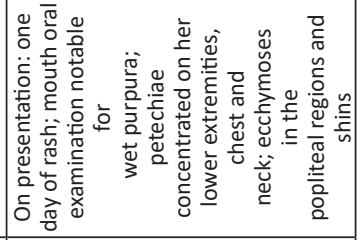 \\
\hline 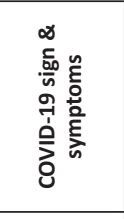 & 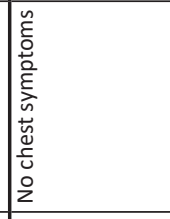 & 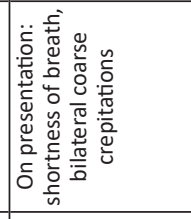 & 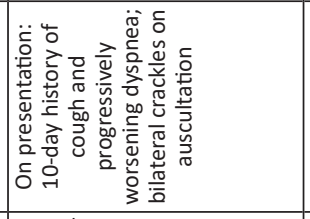 & 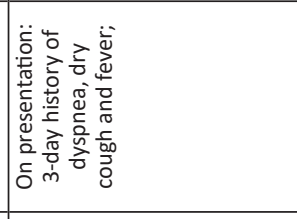 & 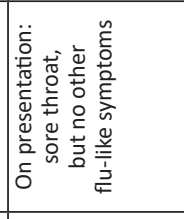 & 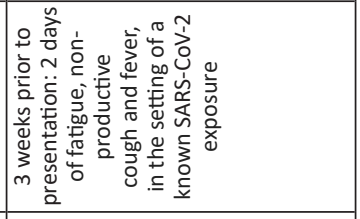 \\
\hline 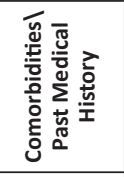 & 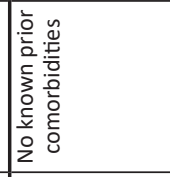 & 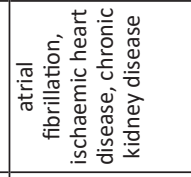 & 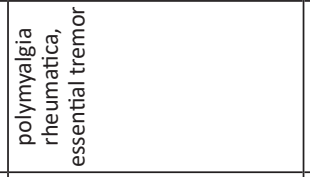 & 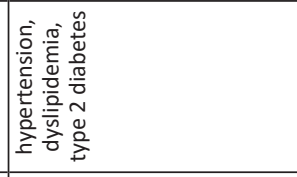 & 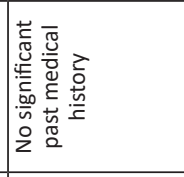 & 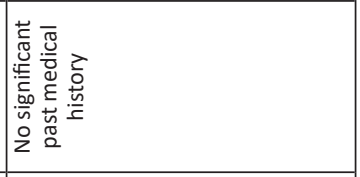 \\
\hline 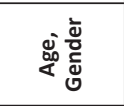 & 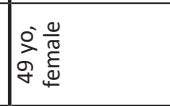 & 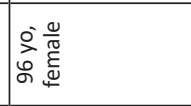 & 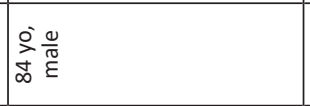 & 产学 & 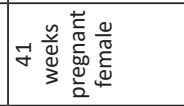 & 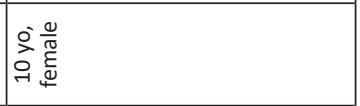 \\
\hline 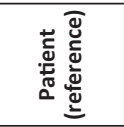 & 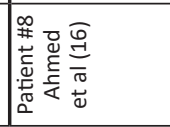 & 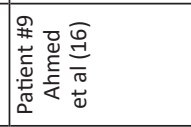 & 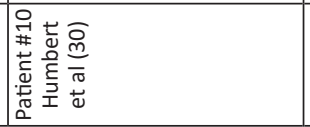 & 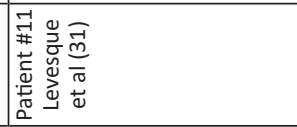 & 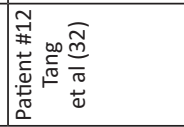 & 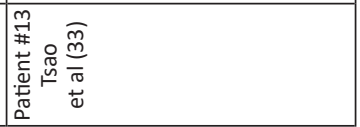 \\
\hline
\end{tabular}




\begin{tabular}{|c|c|c|c|}
\hline 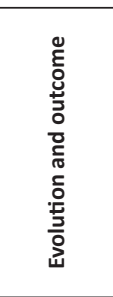 & 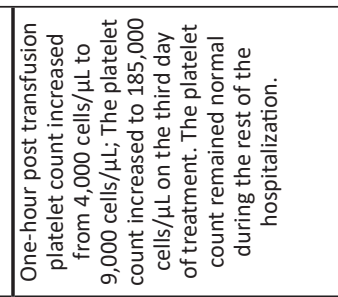 & 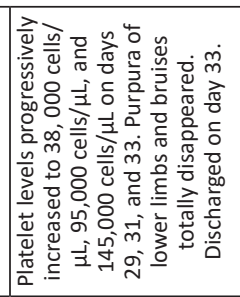 & 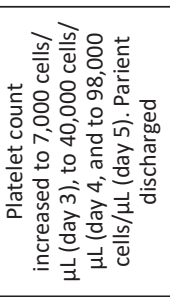 \\
\hline 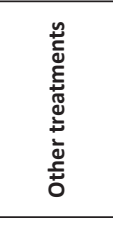 & 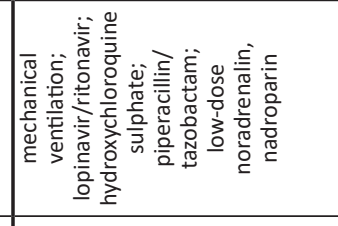 & 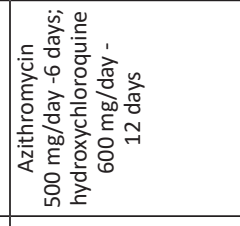 & 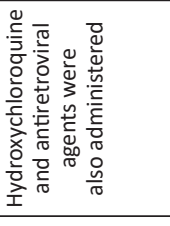 \\
\hline 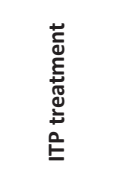 & 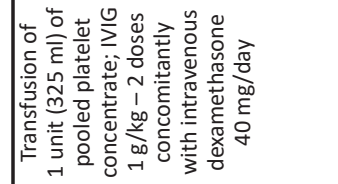 & 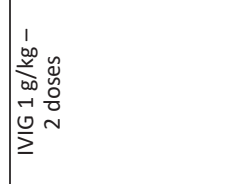 & 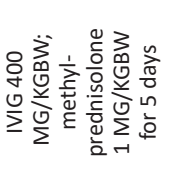 \\
\hline 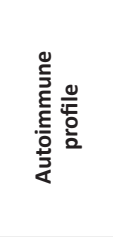 & 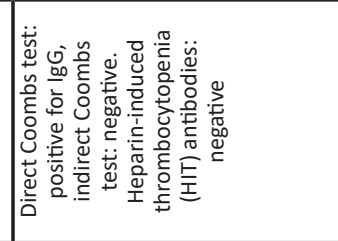 & 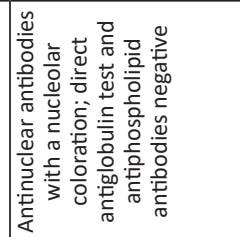 & 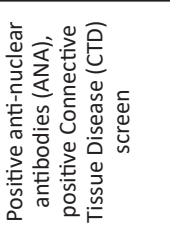 \\
\hline 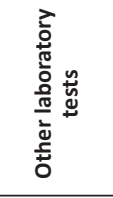 & 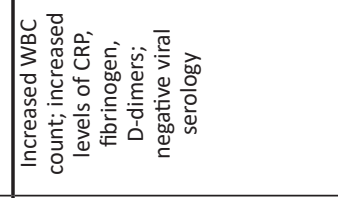 & 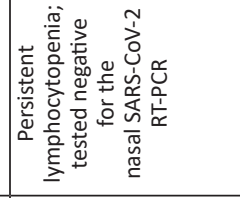 & 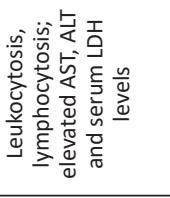 \\
\hline 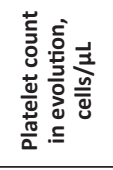 & 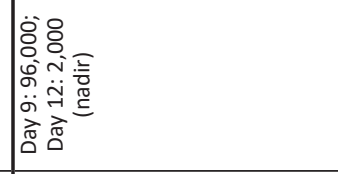 & 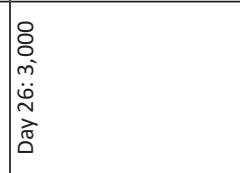 & 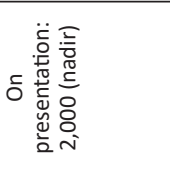 \\
\hline 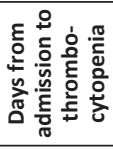 & 㐫 & 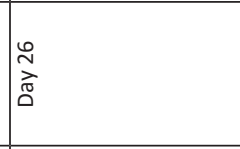 & 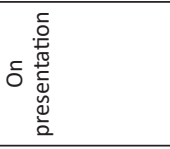 \\
\hline 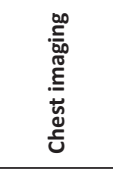 & 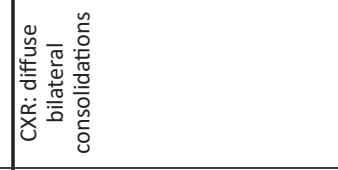 & 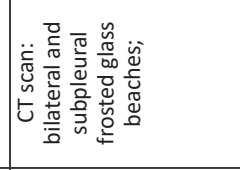 & 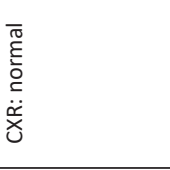 \\
\hline 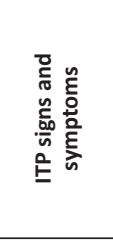 & 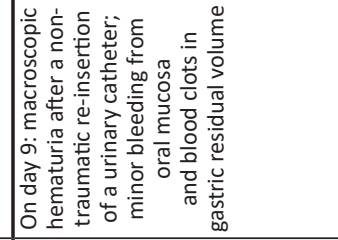 & 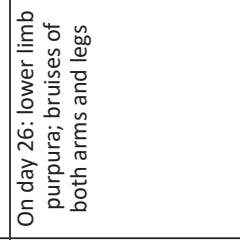 & 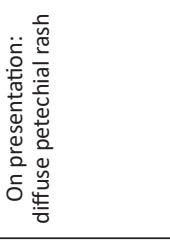 \\
\hline 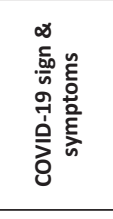 & 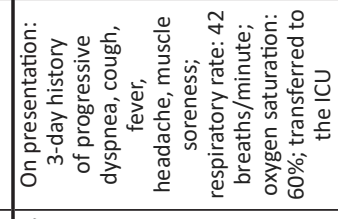 & 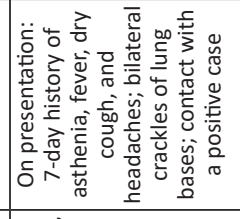 & 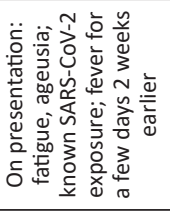 \\
\hline 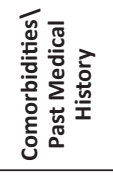 & 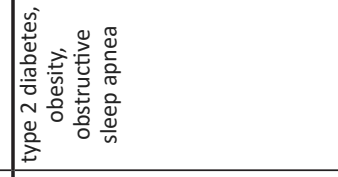 & 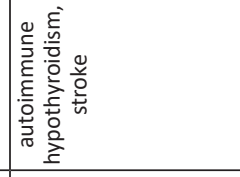 & 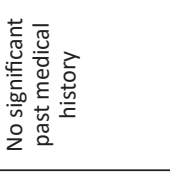 \\
\hline 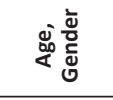 & 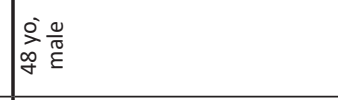 & 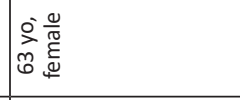 & 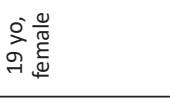 \\
\hline 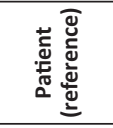 & 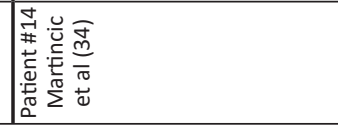 & 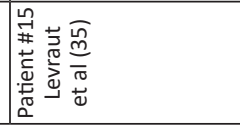 & 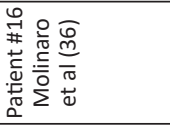 \\
\hline
\end{tabular}


and platelet transfusions. In several of the cases documented, intravenous immunoglobulin administration resulted in a favorable response relatively fast (Patient \#2, \#7, \#8, \#13, \#15) $(16,27,33,35)$. IVIG was administered for Patient \#3 due to active bleeding and the relative contraindication of glucocorticoid medication, which may interact with his prior treatment (somatostatin analog therapy for the neuroendocrine tumor), and for Patient \#4 due to dexamethasone treatment failure (28).

Glucocorticoids comprise the main therapy of ITP (40). However, glucocorticoids are considered unsafe for patients with COVID-19 infection as they inhibit immune responses and clearance of the novel coronavirus $(9,42)$.

Thrombopoietin receptor agonists have made a significant contribution to the treatment in patients with immune thrombocytopenia, which are refractory to first-line agents. About $30 \%$ of patients have shown a steady rise in platelet counts after treatment (43). Because TPO-RA therapy has demonstrated that the risk of venous thromboembolism has in- creased in selected patients, it should be used judiciously in COVID-19 infection (44).

\section{CONCLUSIONS}

Several hematological abnormalities that might lead to life-threatening bleeding complications were identified in COVID-19. Such manifestations must be included in the clinical evaluation of patients infected by SARS-CoV-2. Since the outbreak of the pandemic, there were several case reports of immunemediated thrombocytopenia linked to COVID-19. Thrombocytopenia may be attributed to different reasons and promptly diagnosis of the immunological cause is essential, so that proper immunosuppression may be initiated on time. Failure of timely recognition may eventually result in serious complications. The management of ITP should be decided upon the balance of the bleeding risk due to immune thrombocytopenia versus the prospective complication of COVID-19-infection due to immunosuppressive treatment.

Conflict of interest: none declared Financial support: none declared

\section{REFERENCES}

1. Zhu N, Zhang D, Wang W, Li X, Yang B, Song J, Zhao X, et al.; China Novel Coronavirus Investigating and Research Team. A Novel Coronavirus from Patients with Pneumonia in China, 2019. N Engl J Med. 2020 Feb 20;382(8):727-733.

2. Cucinotta D, Vanelli M. WHO Declares COVID-19 a Pandemic. Acta Biomed. 2020 Mar 19;91(1):157-160.

3. World Health Organization. Weekly epidemiological update on COVID-19 - 25 May 2021. Available at: https://www.who.int/ publications/m/item/weekly-epidemiological-update-on-covid-19--25-may-2021.

4. Hu B, Guo H, Zhou P, Shi ZL. Characteristics of SARS-CoV-2 and COVID-19. Nat Rev Microbiol. 2021 Mar;19(3):141-154.

5. Guan WJ, Ni ZY, Hu Y, Liang WH, Ou CQ, He JX, et al.; China Medical Treatment Expert Group for Covid-19. Clinical Characteristics of Coronavirus Disease 2019 in China. N Engl J Med. 2020 Apr 30;382(18):1708-1720.

6. Wang D, Hu B, Hu C, Zhu F, Liu X, Zhang J, Wang B, Xiang H, et al. Clinical Characteristics of 138 Hospitalized Patients With 2019 Novel Coronavirus-Infected Pneumonia in Wuhan, China. JAMA. 2020 Mar 17;323(11):1061-1069.

7. Vaira LA, Salzano G, Deiana G, De Riu G. Anosmia and Ageusia: Common Findings in COVID-19 Patients. Laryngoscope. 2020 Jul;130(7):1787.

8. Hu B, Guo H, Zhou P, Shi ZL. Characteristics of SARS-CoV-2 and COVID-19. Nat Rev Microbiol. 2021 Mar;19(3):141-154.

9. Zhang Y, Zeng X, Jiao Y, Li Z, Liu Q, Ye J, Yang M. Mechanisms involved in the development of thrombocytopenia in patients with COVID-19. Thromb Res. 2020 Sep;193:110-115.

10. Ehrenfeld M, Tincani A, Andreoli L, Cattalini M, Greenbaum A, Kanduc D, Alijotas-Reig J, Zinserling V, Semenova N, Amital H, Shoenfeld Y. COVID-19 and autoimmunity. Autoimmun Rev. 2020 Aug;19(8):102597.

11. Taherifard E, Taherifard E, Movahed H, Mousavi MR. Hematologic autoimmune disorders in the course of COVID-19: a systematic review of reported cases. Hematology. 2021 Dec;26(1):225-239.

12. Rodríguez Y, Novelli L, Rojas M, De Santis M, Acosta-Ampudia Y, Monsalve DM, et al. Autoinflammatory and autoimmune conditions at the crossroad of COVID-19. J Autoimmun. 2020 Nov;114:102506.

13. Sahu KK, Borogovac A, Cerny J. COVID-19 related immune hemolysis and thrombocytopenia. J Med Virol. 2021 Feb; 93(2):1164-1170.

14. Xu P, Zhou Q, Xu J. Mechanism of thrombocytopenia in COVID-19 patients. Ann Hematol. 2020 Jun;99(6):1205-1208.

15. Mehta P, McAuley DF, Brown M, Sanchez E, Tattersall RS, Manson JJ; HLH Across Speciality Collaboration, UK. COVID-19: consider cytokine storm syndromes and immunosuppression. Lancet. 2020 Mar 28;395(10229):1033-1034.

16. Ahmed MZ, Khakwani M, Venkatadasari I, Horgan C, Giles H, Jobanputra S, Lokare A, Ewing J, Paneesha S, Murthy V. Thrombocytopenia as an initial manifestation of COVID-19; case series and literature review. Br J Haematol. 2020 Jun; 189(6):1057-1058.

17. Rodeghiero F, Stasi R, Gernsheimer T, Michel M, Provan D, Arnold $\mathrm{DM}$, et al. Standardization of terminology, definitions and outcome criteria in immune thrombocytopenic purpura of adults and children: report from an international working group. Blood. 2009 Mar 12;113(11):2386-93.

18. Cines DB, Bussel JB, Liebman HA, Luning Prak ET. The ITP syndrome: pathogenic and clinical diversity. Blood. 2009 Jun 25;113(26):6511-21.

19. Swinkels M, Rijkers M, Voorberg J, Vidarsson G, Leebeek FWG, Jansen AJG. Emerging Concepts in Immune Thrombocytopenia. Front Immunol. 2018 Apr 30;9:880. 
20. Rose NR. Negative selection, epitope mimicry and autoimmunity. Curr Opin Immunol. 2017 Dec;49:51-55.

21. Chen N, Zhou M, Dong X, Qu J, Gong F, Han Y, Qiu Y, Wang J, Liu Y, Wei Y, Xia J, Yu T, Zhang X, Zhang L. Epidemiological and clinical characteristics of 99 cases of 2019 novel coronavirus pneumonia in Wuhan, China: a descriptive study. Lancet. 2020 Feb 15;395(10223):507-513.

22. Guan WJ, Ni ZY, Hu Y, Liang WH, Ou CQ, He JX, Liu L, Shan H, Lei $\mathrm{CL}$, et al.; China Medical Treatment Expert Group for Covid-19. Clinical Characteristics of Coronavirus Disease 2019 in China. N Engl J Med. 2020 Apr 30;382(18):1708-1720.

23. Lippi G, Plebani M, Henry BM. Thrombocytopenia is associated with severe coronavirus disease 2019 (COVID-19) infections: A meta-analysis. Clin Chim Acta. 2020 Jul;506:145-148.

24. Huang C, Wang Y, Li X, Ren L, Zhao J, Hu Y, Zhang L, Fan G, Xu J, et al. Clinical features of patients infected with 2019 novel coronavirus in Wuhan, China. Lancet. 2020 Feb 15; 395(10223):497-506.

25. Tang N, Li D, Wang X, Sun Z. Abnormal coagulation parameters are associated with poor prognosis in patients with novel coronavirus pneumonia. J Thromb Haemost. 2020 Apr;18(4):844-847.

26. Zulfiqar AA, Lorenzo-Villalba N, Hassler P, Andrès E. Immune Thrombocytopenic Purpura in a Patient with Covid-19. N Engl J Med. 2020 Apr 30;382(18):e43.

27. Murt A, Eskazan AE, YIImaz U, Ozkan T, Ar MC. COVID-19 presenting with immune thrombocytopenia: A case report and review of the literature. J Med Virol. 2021 Jan;93(1):43-45.

28. Bomhof G, Mutsaers PGNJ, Leebeek FWG, Te Boekhorst PAW, Hofland J, Croles FN, Jansen AJG. COVID-19-associated immune thrombocytopenia. Br J Haematol. 2020 Jul;190(2):e61-e64.

29. Deruelle E, Ben Hadj Salem O, Sep Hieng S, Pichereau C, Outin H, Jamme M. Immune thrombocytopenia in a patient with COVID-19. Int J Hematol. 2020 Dec;112(6):883-888.

30. Humbert S, Razanamahery J, Payet-Revest C, Bouiller K, Chirouze C. COVID-19 as a cause of immune thrombocytopenia. Med Mal Infect. 2020 Aug;50(5):459-460.

31. Lévesque V, Millaire É, Corsilli D, Rioux-Massé B, Carrier FM. Severe immune thrombocytopenic purpura in critical COVID-19. Int J Hematol. 2020 Nov;112(5):746-750.

32. Tang MW, Nur E, Biemond BJ. Immune thrombocytopenia due to COVID-19 during pregnancy. Am J Hematol. 2020

Aug;95(8):E191-E192.
33. Tsao HS, Chason HM, Fearon DM. Immune Thrombocytopenia (ITP) in a Pediatric Patient Positive for SARS-CoV-2. Pediatrics. 2020 Aug;146(2):e20201419.

34. Martincic Z, Skopec B, Rener K, Mavric M, Vovko T, Jereb M, Lukic M. Severe immune thrombocytopenia in a critically ill COVID-19 patient. Int J Infect Dis. 2020 Oct;99:269-271.

35. Levraut M, Ottavi M, Lechtman S, Mondain V, Jeandel PY. Immune thrombocytopenic purpura after COVID-19 infection. Int J Lab Hematol. 2021 Feb;43(1):e28-e30.

36. Molinaro E, Novara E, Bonometti R, Sacchi MC, Stobbione P, Lauritano EC, Boverio R. Isolated immune thrombocytopenic purpura in a young adult COVID-19 patient. Eur Rev Med Pharmacol Sci. 2020 Oct;24(20):10850-10852.

37. Chen W, Li Z, Yang B, Wang P, Zhou Q, Zhang Z, Zhu J, Chen X, Yang $P$, Zhou $\mathrm{H}$. Delayed-phase thrombocytopenia in patients with coronavirus disease 2019 (COVID-19). Br J Haematol. 2020 Jul;190(2):179-184.

38. Liu Y, Sun W, Guo Y, Chen L, Zhang L, Zhao S, Long D, Yu L. Association between platelet parameters and mortality in coronavirus disease 2019: Retrospective cohort study. Platelets. 2020 May 18;31(4):490-496.

39. Neunert C, Lim W, Crowther M, Cohen A, Solberg L Jr, Crowther MA; American Society of Hematology. The American Society of Hematology 2011 evidence-based practice guideline for immune thrombocytopenia. Blood. 2011 Apr 21;117(16):4190-207.

40. Cooper N, Ghanima W. Immune Thrombocytopenia. N Engl J Med. 2019 Sep 5;381(10):945-955.

41. Cao W, Liu X, Bai T, Fan H, Hong K, Song H, Han Y, Lin L, Ruan L, $\mathrm{Li}$ T. High-Dose Intravenous Immunoglobulin as a Therapeutic Option for Deteriorating Patients With Coronavirus Disease 2019. Open Forum Infect Dis. 2020 Mar 21;7(3):ofaa102.

42. Russell CD, Millar JE, Baillie JK. Clinical evidence does not support corticosteroid treatment for 2019-nCoV lung injury. Lancet. 2020 Feb 15;395(10223):473-475.

43. Kapur R, Aslam R, Speck ER, Rebetz JM, Semple JW. Thrombopoietin receptor agonist (TPO-RA) treatment raises platelet counts and reduces anti-platelet antibody levels in mice with immune thrombocytopenia (ITP). Platelets. 2020;31(3):399-402.

44. Jansen AJ, Swart RM, te Boekhorst PA. Thrombopoietin-receptor agonists for immune thrombocytopenia. N Engl J Med. 2011 Dec 8;365(23):2240-1. 